\title{
El futuro de la
}

educación en una sociedad del conocimiento: el argumento radical en defensa de un currículo centrado en materias*

The Future of Education in a Knowledge Society: The Radical Case for a Subject-Based Curriculum

Michael F. D. Young**

Traductores:

Gustavo Parra***

Laura Macias*4

* Artículo original: Young, Michael. (2010) The future of education in a knowledge society: The radical case for a subject-based curriculum. Pacific-Asian Education, Journal of the Pacific Circle Consortium for Education, vol. 22, N. 1, pp. 21-32.

* Catedrático de Sociología de la Educación en el Institut of Education de la Universidad de Londres, Gran Bretaña. Correo electrónico: m.young@ioe.ac.uk

*** Profesor de la Universidad Pedagógica Nacional, Estudiante de maestría en Educación, e integrante del Grupo de Historia de la Práctica Pedagógica en Colombia. Correo electrónico: gaparral@pedagogica.edu.co

*4 Profesora del Instituto Pedagógico Nacional, Licenciada en Psicología y Pedagogía, integrante del semillero de investigadores del Grupo de Historia de la Práctica Pedagógica en Colombia. Correo electrónico: laurismaxias@hotmail.com

Fecha de recepción: mayo 17 de 2016 Fecha de aprobación: mayo 30 de 2016

\section{Fecha de aprobación: mayo 30 de 2016}

Traducción Pedagogía y Saberes No. 45
Universidad Pedagógica Nacional Facultad de Educación. 2016. pp. 79-88 


\section{Introducción}

$\mathrm{M}$ ucho se escribe en las políticas educativas actuales sobre la preparación de los estudiantes para una sociedad del conocimiento y el importante rol desempeñado por la educación. Esas políticas, sin embargo, dicen muy poco sobre el papel mismo del conocimiento en esta (Young, 2009a). En otras palabras, ¿qué es lo que importa que nuestros jóvenes sepan? Aún más preocupante que eso, muchas políticas actuales casi que de manera sistemática ignoran o marginalizan la cuestión del conocimiento. El énfasis, invariablemente, recae sobre los aprendices, sus diferentes estilos de aprendizaje y sus intereses, sobre resultados de aprendizaje medibles y competencias y como volver el currículo relevante para sus experiencias y su futura empleabilidad. El conocimiento, de cierta forma, se percibe como incuestionable, o como algo que puede adecuarse a nuestras metas políticas (Young, 2010).

No sería exagerado decir que, en el caso de Inglaterra, Escocia y de algunos otros países europeos, las recientes reformas curriculares están llevando a una reducción o incluso un "vaciamiento de contenido", especialmente para aquellos que no están teniendo éxito en la escuela (Yates y Young, 2010). Con frecuencia, esas reformas son bien intencionadas y tienen objetivos progresistas. Enfatizan en la apertura del acceso, mayor participación y promoción de la inclusión social. Eso vuelve difícil cuestionarlas sin parecer conservador y elitista.

En este artículo quiero argumentar que, si vamos a darle un sentido serio a la importancia de la educación en una sociedad del conocimiento, es necesario volver la cuestión del conocimiento nuestra preocupación central y eso involucra el desarrollo de un abordaje del currículo basado en el conocimiento y en las materias, no en el aprendiz, como presume la ortodoxia actual. Además, argumentaría que esa es la opción "radical" — no como afirman algunos, la opción conservadora- siempre que tengamos claro lo que entendemos como conocimiento. Uso aquí la palabra radical para referirme a la cuestión clave que muchos países enfrentan hoy: la persistencia de desigualdades sociales en la educación. Prefiero la palabra radical respecto a alternativas como progresista y crítica. Mientras que la primera tiene una asociación próxima y, en mi opinión, poco conveniente con pedagogías centradas en el aprendiz y con énfasis en el "aprender de la experiencia", la segunda, aunque forme parte de una herencia intelectual mucho más amplia, que se remonta a Kant y a la Ilustración del siglo XVIII, ha estado relacionada, en los estudios educacionales, con la retórica hueca de mucho de lo que pasa por pedagogía crítica.

El resto del texto trata acerca de cómo pensamos sobre el currículo; consta de dos partes. En la primera me refiero al ejemplo de las reformas de 2008 del Currículo Nacional en Inglaterra, el cual describo como adopción de un abordaje "instrumentalista". Más adelante explicaré lo que quiero decir con eso. Argumentaré que, en lugar de ello, necesitamos concebir el currículo no como un instrumento para alcanzar objetivos tales como "contribuir a la economía" o "motivar aprendices descontentos", sino como intrínseco al motivo por el que al fin de cuentas tenemos escuelas. La segunda parte del artículo desplaza la mirada del currículo a las escuelas y sugiere cómo las disciplinas o materias escolares ${ }^{1}$ pueden considerarse como el recurso más importante para el trabajo de los profesores y de los alumnos en la escuela.

En la sección final, trato dos de los argumentos más fuertes presentados en contra de un currículo centrado en materias, o más generalmente, centrado en el conocimiento. El primer argumento es que cualquier forma de currículo centrado en materias continuará discriminando a los menos favorecidos y, principalmente, a los alumnos de la clase trabajadora y de las minorías étnicas. Ese asunto tiene una urgencia particular en el Reino Unido en este momento. Un currículo tradicional centrado en disciplinas es fuertemente avalado por el conservador secretario de Estado para la Educación, Michael Gove. Un mes antes de la elección general él dijo que era un adepto convencido de un currículo tradicionalista y que creía que la mayoría de los padres querían que sus hijos "se sentaran en filas, aprendieran sobre reyes y reinas, leyeran obras importantes, hicieran cálculos mentales, comenzaran a aprender álgebra a los 11 años y aprendieran idiomas extranjeros" (Gove, 2009). Es importante diferenciar entre la visión de currículo de Gove de un currículo centrado en disciplinas y la visión de currículo que defiendo. Haré esto de dos maneras: en términos de sus diferentes concepciones de conocimiento, y en términos de los distintos supuestos que se establecen con respecto a las relaciones de los aprendices con el conocimiento.

1 Nota de los traductores. En español, la palabra subject empleada en relación con la escuela y el currículo puede traducirse como materias o disciplinas escolares. En el texto, aunque se opte principalmente por el primer término, también se emplea el segundo como sinónimo, y en un sentido diferente a la disciplina como conjunto de normas o como el régimen y la organización de la vida escolar. 
El modelo tradicional trata el conocimiento como dado y como algo que los estudiantes tienen que cumplir. En contraste, aunque el modelo que defiendo también trate el conocimiento como exterior a los aprendices, reconoce que esa exterioridad no está dada, sino que tiene una base social e histórica. También distingo el modelo que defiendo del tradicional, por sus diferentes modos de relación con los aprendices y, por tanto, sus implicaciones diferentes para la pedagogía y lo que hacen profesores y alumnos. A la visión planteada por Gove me referiré como un "currículo basado en la conformidad", y la otra como un "currículo basado en el compromiso".

Lo que los dos modelos tienen en común y cómo se sitúan en contraste con el modelo instrumentalista que sustenta las reformas de 2008 en Inglaterra es que ambos parten del conocimiento y no del aprendiz ni de los contextos que estos enfrentan, como lo sugieren currículos proyectados para adecuarse al futuro empleo del alumno.

El segundo argumento contra un currículo basado en materias, el cual trataré más brevemente al final, es la afirmación de que está en desacuerdo con lo que con frecuencia se proclama como una tendencia global para la des-diferenciación; en otras palabras, el debilitamiento de las fronteras entre ocupaciones y dominios de conocimiento.

\section{Las reformas de 2008 en Inglaterra: currículos instrumentalistas y sus problemas}

Las políticas curriculares se desarrollan, inevitablemente, en contextos sociales, políticos y económicos. Mi argumento es que en la última década, bajo presiones globales bien conocidas, los diseñadores de currículo en el Reino Unido dieron demasiada importancia a esos contextos, en dos sentidos. En primer lugar, respondieron a la presión del gobierno, de contribuir a la solución de problemas sociales, como el desempleo. En segundo lugar, también respondieron a lo que percibían como necesidades e intereses de los aprendices, especialmente de aquellos con bajo rendimiento en la escuela o que la abandonaban pronto.

En consecuencia, las propuestas ignoraron, o por lo menos dejaron en segundo plano, el papel educativo fundamental del currículo, que se deriva tanto del propósito de las escuelas como de lo que ellas pueden o no hacer. Aunque no debemos olvidar el contexto más amplio, las elecciones curriculares deben ser tratadas por lo que son: maneras alter- nativas de promover el desarrollo intelectual de los jóvenes. Cuanto más nos enfocamos en la posibilidad de un currículo reformado para resolver problemas sociales o económicos, tanto menos probable es que esos problemas sean tratados en sus orígenes, que no se encuentran en la escuela.

El ex primer ministro Tony Blair una vez declaró: "La educación es la mejor política económica que tenemos". Eso decía mucho, por extensión, de sus políticas económicas. No obstante, también representa el tipo de instrumentalización que ha infestado las políticas educacionales en Inglaterra en los últimos treinta años; trata de lo que los políticos esperan que la educación pueda hacer "como un medio" y no de su razón de ser "como un fin". Es como si aquellas cuestiones sobre las finalidades de la educación fuesen demasiado filosóficas y abstractas para los formuladores de política y los políticos. Lamentablemente, los filósofos de la educación tienden a exacerbar el problema al invocar ideas como "bienestar" (White, 2007). Desde luego, el bienestar humano es un objetivo importante para todas las sociedades; con todo, es tanto un objetivo para familias y comunidades como para las escuelas, y dice poco sobre el papel específico de estas últimas.

Las principales prioridades de las reformas de 2008 fueron dar menos peso al contenido de las disciplinas y más a los temas que atraviesan un amplio espectro de disciplinas y buscar maneras para personalizar el currículo, relacionándolo más directamente con el conocimiento y las experiencias cotidianas del alumno. Los diseñadores de currículo parten de dos problemas que ciertamente no ocurren solo en Inglaterra: un currículo "sobrecargado" y demasiados alumnos descontentos. Las reformas intentaban vincular los dos para explicar el fracaso de las escuelas en motivar a una proporción significativa de estudiantes. El currículo reformado enfatizaba en su flexibilidad y su relevancia para la experiencia que los estudiantes llevan a la escuela. En otras palabras, el currículo era visto como un instrumento para motivar a los estudiantes a aprender.

\section{¿Por qué eso es un problema?}

Mi argumento se basa en un corto texto de Tim Oates (2009). Un abordaje instrumentalista del currículo distorsiona lo que cualquier currículo puede hacer y confunde dos ideas educacionales crucialmente distintas. La primera dice, respecto al currículo, que se refiere al conocimiento que un país considera importante que esté al alcance de los estudiantes. La segunda, dice que, en contraste, la pedagogía se 
refiere a las actividades de los profesores para motivar a los alumnos y ayudarlos a comprometerse con el currículo y volverlo significativo.

Es necesario que currículo y pedagogía, sugiero, sean vistos como conceptualmente distintos. Se refieren a responsabilidades distintas de diseñadores de currículo y profesores, y los unos dependen de los otros. Mientras que los profesores no pueden crear ellos mismos un currículo, sino que necesitan de este para guiarlos en lo que deben enseñar, los diseñadores curriculares apenas pueden estipular los conceptos importantes a los cuales los alumnos requieren tener acceso. Los formuladores de currículo cuentan con los profesores para motivar a los estudiantes y transformar esos conceptos en una realidad para los alumnos.

Las tentativas de incluir las experiencias de los alumnos en un currículo "más motivador" oscurecen la distinción currículo/pedagogía y los papeles muy diferentes de formuladores de currículo y profesores. Como la mayoría de los profesores saben bien, ellos deben tener en cuenta las experiencias y el conocimiento anterior que los alumnos llevan a la escuela y lo que los motiva inicialmente. Estos son algunos de los recursos con que cuentan para movilizar a los estudiantes, y constituye la base para que ellos se vuelvan aprendices activos. Eso, no obstante, es bastante diferente de incluir esas experiencias en el currículo.

Quiero mencionar otros dos problemas que pueden surgir de una visión instrumentalista del currículo. Ambos se relacionan con el oscurecimiento de la distinción entre currículo y pedagogía y, por eso, ambos conducen directamente a una discusión sobre el papel que las disciplinas tienen en el currículo. En primer lugar, una visión instrumentalista del currículo puede llevar a un efecto perturbador, demostrado vivamente en las directrices de la Qualifications and Curriculum Development Agency (QCDA) (Agencia de Cualificación y Desarrollo del Currículo) (véase http://www.qcda.gov.uk/curriculum/36. aspx). Específicamente me refiero a la proliferación de directrices particulares para profesores. Aunque no estén obligados estatutariamente a adoptarlas, la naturaleza autoritaria de sus orígenes en la QCDA, junto con los vínculos con especificaciones de las disciplinas en que se basan los exámenes, las hacen difíciles de ignorar. Parece que las directrices presumen que la solución para la falta de motivación de los estudiantes es dar más orientación curricular para los profesores, en lugar de fortalecer y apoyar su conocimiento pedagógico y de la materia y, en consecuencia, su profesionalismo.
En un contexto político más amplio, en el cual se da mucha importancia a las notas de los alumnos y a los resultados de pruebas en las que las escuelas pueden ser jerarquizadas nacionalmente según el número de alumnos que reciben certificados, no es exagerado sostener que el currículo se está tornando cada vez más en una forma de rendir cuentas en lugar de una guía para los profesores. Dos ejemplos contrastantes de especificaciones curriculares ilustran esa cuestión. Uno vino de la QCDA y estaba siendo usado por una escuela del gobierno; tenía unas diez o doce páginas. El otro, de un Consejo de Exámenes (Examination Board), estaba siendo usado por una escuela particular paga y ocupaba una página y media. Ambos trataban de la cuestión de la calidad. Sin embargo, tenían ideas bien diferentes tanto en lo que respecta al profesionalismo del profesor, como a la distinción entre currículo y pedagogía, así como hasta el punto en que se podía confiar en los profesores.

El segundo problema que surge cuando se trata el currículo como un "instrumento" es que se hace posible que los gobiernos aduzcan que problemas económicos o sociales se pueden "solucionar" con cambios en el currículo. No niego que el currículo debe estar siempre abierto al debate democrático. Sin embargo, a no ser que exigencias políticas de los gobiernos tengan que enfrentar criterios educacionales explícitos de los formuladores de currículo sobre lo que este puede hacer, hay un peligro de omitir la finalidad más fundamental de la educación escolar, que es llevar a los alumnos más allá de sus experiencias por formas a las que ellos tendrían difícilmente acceso en casa. Ciertamente para eso son las escuelas.

Resumiendo mi argumentación hasta ahora: en primer lugar, el currículo necesita verse con una finalidad propia, el desarrollo intelectual de los estudiantes. No debe tratarse como un medio para motivar a los estudiantes o para solucionar problemas sociales.

En segundo lugar, el desarrollo intelectual es un proceso basado en conceptos, y no en contenidos o habilidades. Eso significa que el currículo debe basarse en conceptos. Sin embargo, los conceptos son siempre sobre alguna cosa, implican unos contenidos y no otros. El contenido, por tanto, es importante, no como datos por memorizar, como en el currículo antiguo, sino porque sin él los estudiantes no pueden adquirir conceptos y, por ende, no desenvolverán su comprensión ni progresarán en su aprendizaje.

En tercer lugar, debe distinguirse entre currículo y pedagogía, una vez que se relacionan de modo diferente con el conocimiento escolar y con el conocimiento cotidiano que los alumnos llevan a la escuela. 
El currículo debe excluir el conocimiento cotidiano de los estudiantes, al mismo tiempo que ese conocimiento es un recurso para el trabajo pedagógico de los profesores. Los estudiantes no van a la escuela para aprender lo que ya saben.

En cuarto lugar, son los profesores con su pedagogía, y no los diseñadores de currículo, quienes se sirven de la cotidianeidad de los alumnos para ayudarlos a comprometerse con los conceptos definidos en el currículo y percibir su relevancia.

Finalmente, el conocimiento incluido en el currículo no debe basarse en el conocimiento especializado desarrollado por comunidades de investigadores. Ese proceso ha sido descrito como recontextualización del currículo (Barnett, 2006). Sin embargo, esas comunidades de investigación no se involucran con las escuelas; por consiguiente, el currículo no puede establecer cómo se accede a ese conocimiento. Ese nuevo proceso de "recontextualización" será específico para cada escuela y para la comunidad en que se localiza, y se basa en el conocimiento profesional de los profesores. ¿Por qué entonces el currículo debe centrarse en disciplinas? Ese es el tema de la segunda parte de este artículo.

\section{Disciplinas, el currículo y los objetivos de la escolarización}

En esta sección, quiero desplazar la mirada del currículo para la escuela, y de los formuladores de currículo para los profesores de las disciplinas. Aquí me valgo de la obra del sociólogo y filósofo francés Bernard Charlot (2009), quien inicia su discusión estableciendo lo que es la escuela y el tipo de lugar que la caracteriza. Resaltaré cinco aspectos relacionados en la elaboración de su argumento.

Las escuelas son lugares donde el mundo es tratado como un "objeto de pensamiento" y no como un "lugar de experiencia". Disciplinas como historia, geografía y física son las herramientas que los profesores tienen para ayudar a los alumnos a pasar de la experiencia a lo que el psicólogo ruso Vygotsky refirió como "formas más elevadas de pensamiento". Las disciplinas reúnen "objetos de pensamiento" como conjuntos de "conceptos" sistemáticamente relacionados.

Algunas veces, esos conceptos tienen referentes fuera de la escuela, en el ambiente de vida del alumno, en una ciudad como Londres por ejemplo. No obstante, las relaciones de los alumnos con Londres como un "concepto" deben ser diferentes de la relación con su "experiencia" de Londres como el lugar donde viven.
Es importante que los alumnos no confundan el Londres del cual habla el profesor de geografía con su experiencia en Londres como lugar donde viven. Hasta cierto punto, es la misma ciudad, pero la relación del alumno con ella, en los dos casos, no es la misma. El Londres donde viven es un "lugar de experiencia". Londres como ejemplo de una ciudad es un "objeto de pensamiento" o un "concepto".

Si los alumnos no consiguen captar la diferencia entre pensar Londres como un ejemplo del concepto geográfico de ciudad y su experiencia de vivir allí, tendrán problemas para aprender geografía y, por analogía, cualquier disciplina escolar que busque llevarlos más allá de su experiencia. Por ejemplo, la profesora tal vez pregunte a la clase cuáles son las funciones de la ciudad de Londres. Eso implica que piensen en la ciudad en relación con el Gobierno y el comercio, y no solo que describan cómo ellos, sus padres y sus amigos experimentan la vida en la ciudad.

Ese argumento puede expresarse de otra forma. Los conceptos "teóricos" de disciplinas como la geografía y los conceptos "cotidianos" que constituyen la experiencia que los alumnos llevan a la escuela son distintos y usarlos implica procesos de pensamiento muy diferentes. De nuevo, fue Vygotsky quien primero resaltó esas diferencias. Vale la pena resumirlas.

Los conceptos teóricos se originan en comunidades de especialistas productores de conocimientos, como físicos y geógrafos. Esos conceptos tienen finalidades específicas por el hecho de que nos capacitan para hacer generalizaciones confiables a partir de casos particulares y probarlas. Los conceptos teóricos se relacionan sistemáticamente unos con otros (en materias y disciplinas), y se adquieren consciente y voluntariamente por medio de la pedagogía en las escuelas, facultades y universidades.

En contraste, los conceptos cotidianos los "captamos" inconscientemente en nuestra vida diaria y los adquirimos por medio de la presencia de formas ad hoc para propósitos específicos, relacionados con problemas particulares, en contextos particulares.

Ellos forman el conocimiento que necesitamos para vivir en sociedad. Las disciplinas, por consiguiente, son conjuntos de conceptos teóricos relacionados, tales como la ciudad y los suburbios para geógrafos urbanos y profesores de geografía. También son las formas de organización social que reúnen especialistas en disciplinas y les dan su identidad.

Algunas veces, en geografía como en otras disciplinas, los conceptos curriculares no tienen un referente en el ambiente de vida del alumno. Tales conceptos apenas pertenecen a un mundo específico, constituido por investigadores especialistas 
implicados en desarrollar nuevo conocimiento. Buenos ejemplos son los electrones y átomos en ciencias. Al mismo tiempo, porque fueron estudiados y probados por especialistas, el acceso a ellos es el modo más confiable que tenemos para que el estudiante comprenda mejor.

Charlot (2009) llega a la conclusión de que los profesores poseen dos tareas pedagógicas fundamentales. La primera es ayudar a los estudiantes a manejar su relación entre los conceptos de las diferentes disciplinas que constituyen el currículo y sus referentes en la vida cotidiana. La segunda, presentar a los alumnos conceptos que tienen significados que no derivan de su experiencia ni se relacionan directamente con ella.

Las disciplinas, entonces, tienen dos características como base del diseño curricular. Primero, consisten en conjuntos de conceptos relativamente coherentes que se relacionan distinta y explícitamente entre sí. Disciplinas diferentes tienen reglas para definir las fronteras entre ellas y otras disciplinas y para establecer el modo como sus conceptos se relacionan. Esas reglas variarán según la precisión con que se definen; Bernstein (2000) usa los conceptos "jerárquico" y "segmentado" para distinguir disciplinas como física y literatura.

En segundo lugar, las disciplinas también son "comunidades de especialistas" con historias y tradiciones distintas. Por medio de esas "comunidades", profesores en diferentes escuelas y facultades están ligados unos a otros y a aquellos que están en las universidades produciendo nuevos conocimientos. Cada vez más, profesores de países diferentes también se vinculan mediante periódicos, conferencias e internet.

Dos características distinguen esa visión de disciplina, que está asociada con aquello a lo que me referí como un "currículo de compromiso", de la visión tradicionalista de disciplina asociada a un "currículo de acatamiento". La primera es que las disciplinas son entidades históricas dinámicas que cambian con el tiempo, en parte por desarrollo interno gracias a los especialistas, en parte por presiones políticas externas y otras presiones. En contraste con la visión tradicional de disciplinas, ellas no son vistas como parte de algún canon fijo definido por la tradición, con contenidos y métodos inmutables. Eso no significa que sea posible tener una materia o una disciplina sin algún tipo de "canon" de textos, conceptos y métodos acordados. Eso significa que el canon en sí tiene una historia y, aunque no sea fijo e inmutable, tiene una estabilidad, así como una apertura en la que los estudiantes pueden apoyarse al establecer sus identidades.
La segunda diferencia es que, al adquirir conocimientos de las disciplinas, los estudiantes no solo acatan las reglas y los contenidos específicos como si fuesen instrucciones. Al adquirir conocimientos de las disciplinas, están ingresando en aquellas "comunidades de especialistas", cada una con sus diferentes historias, tradiciones y modos de trabajar. Las disciplinas, por tanto, tienen tres papeles en un "currículo de compromiso". El primero es un papel curricular. Las disciplinas garantizan por medio de sus enlaces con el proceso de producción de nuevos conocimientos, que los estudiantes tengan acceso al conocimiento más confiable disponible en campos particulares. El segundo papel es pedagógico. Las disciplinas ofrecen puentes a los aprendices para que pasen de sus "conceptos cotidianos" a los "conceptos teóricos" asociados a ellas. El tercero es un papel generador de identidad para profesores y aprendices. Las disciplinas son cruciales para el sentido de identidad de los profesores como miembros de una profesión.

El conocimiento de la disciplina provee a los profesores la base de su autoridad sobre los alumnos. Para los alumnos, pasar de su mundo cotidiano, en el cual los conceptos son desarrollados por experiencia en relación con problemas que surgen en contextos específicos, al mundo de la escuela, que trata el mundo como un objeto sobre el cual se piensa, puede ser una experiencia amenazadora e incluso extraña. El mundo cotidiano no es como la escuela. No se divide en materias o disciplinas. Ese papel generador de identidad de las disciplinas es particularmente importante para alumnos de lugares desfavorecidos y para sus profesores. Muchos de esos alumnos llegaron a la escuela con poca experiencia de tratar el mundo como más que un conjunto de experiencias, en otras palabras, conceptualmente. Las disciplinas, con sus fronteras para separar aspectos del mundo que fueron probados a lo largo del tiempo, no solo ofrecen la base para analizar y hacer preguntas acerca del mundo, también ofrecen a los estudiantes una base social para un nuevo conjunto de identidades como aprendices. Con las nuevas identidades referentes a las disciplinas, que los estudiantes adquieren por el currículo, sumadas a aquellas con las que vinieron a la escuela, tienen más probabilidades de ser capaces de resistir al sentido de alienación de sus vidas cotidianas fuera de la escuela, o al menos lidiar con él. La escuela puede promover tal capacidad.

Como exprofesor de química y de sociología, tengo alguna idea sobre los conceptos de química, como periodicidad y valencia, y los de sociología, como solidaridad y clase social. Tales conceptos, así como las relaciones entre ellos y el mundo de la vida 
cotidiana, tienen su propia historia en el ámbito de las disciplinas. Ellas son lo que constituye las disciplinas y ofrecen las maneras más poderosas para generalizar más allá de nuestra experiencia del mundo. Es por esa razón que defiendo las disciplinas como base del currículo.

\section{Conclusiones y desafíos}

Desarrollé un argumento defendiendo el papel central de las disciplinas en el currículo escolar e indiqué algunas de las razones por las cuales ese papel ha sido solapado por recientes desarrollos curriculares. Resta, no obstante, una cantidad de cuestiones por tratar.

En muchos países, se está experimentado con un currículo no centrado en disciplinas, sino en temas, líneas de investigación o temas derivados de los intereses de los alumnos, y ha resultado atrayente para profesores y alumnos. Parece resolver las cuestiones de relevancia curricular y del "interés del alumno", además de concebir la experiencia de las disciplinas como una forma de "tiranía cultural". Mi argumento es que inevitablemente faltará coherencia a tales currículos, que explícitamente oscurecen la distinción currículo/pedagogía y que, además, no ofrecen la base necesaria para el progreso de los alumnos. Los criterios para la selección de los aspectos o temas serían, en gran parte, arbitrarios o derivados de las experiencias individuales de los profesores, y no del conocimiento especializado de profesores e investigadores, construido a lo largo del tiempo.

En tal currículo, los profesores tendrían que apoyarse más en su posición de autoridad en la escuela y no en su conocimiento especializado en la disciplina. Además de eso, los estudiantes podrían tener dificultades en establecer sus identidades como aprendices escolares y tenderían o a una lealtad personal a profesores específicos, o a rechazar la autoridad derivada de la posición del profesor, viéndola como burocrática e ilegítima. Este es el comienzo del descontento que frecuentemente los lleva a abandonar la escuela. A pesar de esos problemas, el apoyo a un currículo integrado o temático probablemente no desaparecerá, en especial entre profesores "radicales". Tales currículos parecen ofrecer un modo de superar el problema de la superespecialización. ¿Cómo es que, en un currículo basado en disciplinas, los alumnos adquieren los recursos para "hacer conexiones" y ganar un sentido del mundo como un "todo"? Esa pregunta es importante, pero está más allá del objetivo de este artículo. Entonces, voy a restringirme a algunas breves observaciones. El problema de "conexión" no es fácil de resolver y no hay ninguna evidencia de que la especialización intelectual tenga probabilidad de revertirse. Para las escuelas, supongo, es un problema pedagógico y no curricular. En términos curriculares, no hay alternativa a las disciplinas que se muestre adecuada y que defina los conceptos que queremos que los estudiantes adquieran. No hay principios curriculares generales "de conexión", como yo pensaba (o esperaba) hace algunos años, que diesen algún sentido a la idea de "especialización conectiva" (Young, 1998). Mi respuesta provisoria es que la capacidad de conectar o "atravesar fronteras" puede ser desarrollada por los profesores, y surge de la fuerza de la identidad de los estudiantes relacionada con la disciplina, así como de los problemas que encuentren cuando los conceptos fundamentados en las disciplinas no puedan resolverlos adecuadamente.

Existe un paralelo que precisa ser más explorado entre esa cuestión y la idea expresada por Abbott (2001) y más recientemente por Moore (2011) y Muller (2011) que, en el campo de la producción de conocimiento, "una forma de interdisciplinariedad" es una parte normal del crecimiento del conocimiento. Es una interdisciplinariedad que surge de la apertura y las limitaciones de los campos de conocimiento y no de algún principio exterior impuesto. En el contexto de la escuela, es responsabilidad del profesor de la disciplina monitorear, criticar y, algunas veces, apoyar a aquellos estudiantes que luchan por moverse más allá de las reglas de la materia escolar.

Finalmente, quiero considerar dos objeciones diferentes a mi defensa de un currículo centrado en el conocimiento. La primera es que, a pesar de distinguir entre modelos de currículo de "acatamiento" y de "compromiso", mi modelo de compromiso de un currículo basado en materias difiere muy poco del currículo tradicionalista apoyado por el nuevo secretario de Estado británico. En otras palabras, esto inevitablemente perpetuaría un sistema elitista y desigual y continuaría negando oportunidades de aprendizaje a muchos estudiantes de lugares desfavorecidos. Es un argumento familiar y consistente con la crítica a las materias escolares que hice en mi primer libro, Knowledge and Control (Young, 1971).

Fui llevado a repensar mis ideas anteriores sobre el conocimiento, el currículo y el papel de la escolarización. Eso no significa que ahora no considere cómo las escuelas en las sociedades capitalistas reproducen la clase social u otras desigualdades. Sin embargo, el hecho de que algunos chicos de familias de la clase trabajadora tengan éxito en la escuela, a pesar de sus desventajas culturales, y de que en muchos países las niñas obtienen mejores resultados que los niños (Marrero, 2008), a despecho de la discriminación 
de género en la sociedad, sugiere que el papel de las escuelas y del currículo basado en materias es más complejo como para apenas mantener desigualdades.

En sociedades desiguales como Inglaterra, cualquier currículo mantendrá esas desigualdades. Con todo, la escolarización también representa (o puede representar, dependiendo del currículo) los objetivos universalistas de tratar a todos los alumnos igualmente y no apenas como miembros de clases sociales diferentes, grupos étnicos diferentes o como niños o niñas.

La escolarización común, con el objetivo de maximizar el desarrollo intelectual de todos los estudiantes, puede ser considerada una institución como la ciencia, la democracia y los sindicatos. Ninguna de ellas dio cuenta plenamente de los objetivos que les fueron asociados, pero ninguna es solo un producto del capitalismo o del colonialismo y sus divisiones. La escolarización común surgió, en parte, de las necesidades de un capitalismo industrial en expansión y de las desigualdades de clase social generadas por este. Sin embargo, también fue un producto de la Ilustración del siglo xviII y de los valores de universalismo e igualdad asociados a esta. Las escuelas y el currículo, al igual que instituciones políticas tales como la democracia o los sindicatos, están en constante tensión con su contexto. No son solo productos de este.

Sería ingenuo imaginar que cualquier currículo pudiese superar desigualdades generadas en otro lugar. Sociedades capitalistas, en grados diferentes, siempre producirán desigualdades en educación, salud, vivienda o cualquier servicio público. Por otro lado, un currículo basado en materias tiene un grado de objetividad basado en el presupuesto de que es la manera más confiable que hemos desarrollado para transmitir y adquirir "conocimiento poderoso". Nadie imagina que la creación de nuevo conocimiento podría comenzar con la experiencia o con nuestro día a día. Dicen que Isaac Newton afirmó: "Si he logrado ver más lejos, ha sido porque he subido a hombros de gigantes". Eso también explica la adquisición de conocimiento. Las materias vinculan la adquisición de nuevo conocimiento a su producción. Negar eso en el currículo no es distinto de negar acceso a los retrovirales a africanos con VIH/SIDA con el argumento de que eso muestra desprecio por sus conocimientos locales.

Podemos conectar ese argumento con mi discusión anterior sobre las materias. En general, las familias de clase media dan a sus hijos experiencias para tratar el mundo como "un objeto" o de una manera que presenta algunos paralelos con las materias, no solo como una experiencia, a diferencia de las familias de la clase trabajadora; no sorprende que los primeros estén mejor preparados para un currículo basado en materias. Podemos considerar que se trata de un subsidio de clase media. Al mismo tiempo, las materias, con su secuencia, su ritmo y su selección de contenidos y actividades son lo que más nos llevan, en educación, a ofrecer a los estudiantes acceso a un conocimiento confiable. En otras palabras, idealmente, las disciplinas escolares expresan valores universales que tratan a todos los seres humanos como iguales y no como miembros de diferentes clases sociales, grupos étnicos o niños y niñas. Las escuelas de élite son exitosas por dos razones. La primera, por la capacidad que sus precios elevados les confieren para ser tanto social como intelectualmente selectivas. La segunda, porque tienen recursos para reclutar los mejores profesores especializados en las disciplinas. La falta de profesores bien cualificados en las materias es uno de los mayores motivos por los cuales, en términos relativos, las escuelas estatales no lo hacen tan bien. El debilitamiento de la base de materias del currículo tornará más difícil para los estudiantes distinguir entre "objetos de pensamiento", o conceptos que constituyen un currículo, y sus experiencias. Una razón por la cual el secretario de Estado para la Educación británico está equivocado es que él está aduciendo un objetivo universalista: los profesores de las materias tratan a todos los aprendices de la misma manera; en un contexto no universalista: no todos los estudiantes tienen el mismo acceso a profesores especializados en las diferentes materias.

Una segunda objeción a mi recontextualizado "currículo de compromiso" basado en materias es que este no toma en cuenta las transformaciones globales de la sociedad que ocurrieron y todavía están ocurriendo. Aquí, apenas puedo presentar brevemente mi respuesta; sería necesario otro artículo. El debilitamiento de las fronteras entre las disciplinas escolares y el conocimiento cotidiano con frecuencia se presenta como consistente con transformaciones políticas y económicas asociadas a la globalización. Podemos trazar paralelos con el reciente entusiasmo por un cambio de lo que es tratado como conocimiento Modo 1 para el Modo 2 como la base para un nuevo abordaje de la producción de conocimiento ( $c f$. Gibbons et ál., 1994). Se argumenta, entonces, a favor de un currículo escolar interdisciplinar o temático, por ser más afinado con el mundo "como se está tornando" (Young y Muller, 2010). Mi argumento aquí es que, incluso si esas tendencias globales puedan constituir una previsión precisa de cambio social en las ocupaciones, no tenemos fundamento para 
asumir que estas se aplican a las condiciones para la adquisición de conocimiento confiable, $\mathrm{o}$ a las de su producción.

La producción de conocimiento nuevo por medio de investigación y su adquisición mediante la educación formal son fenómenos relativamente recientes en la historia de la humanidad. Hay un conjunto de trabajos en la sociología del conocimiento que remonta por lo menos al sociólogo francés Emile Durkheim, hace más de un siglo, que explica las condiciones que volvieron eso posible. Durkheim afirma que la diferenciación entre conocimiento y experiencia, y entre conocimiento teórico y conocimiento cotidiano son las condiciones fundamentales para la adquisición y la producción de nuevo conocimiento (Durkheim, 1983 y Young, 2008).

Concluiré citando a Max Weber, el sociólogo alemán cuya carrera transcurrió entre 1890 y 1920 . En el final de su famoso libro, La ética protestante y el espíritu del capitalismo, escribió:

En la civilización occidental, y solamente en la civilización occidental, aparecen fenómenos culturales [y el currículo basado en materias podría ser considerado como uno, aunque él no estuviera refiriéndose a eso] que [como nos gustaría pensar] se encuentran en una línea de desarrollo que tiene significancia y validez universales. (Citado en Kronman, 2007)

Para algunos, eso puede sonar como una forma de neocolonialismo primitivo, intentar extraer un conjunto de ideas de su contexto político e histórico y afirmar su universalidad. Creo que Weber estaba planteando una pregunta con implicaciones muy profundas para los que trabajamos en educación. La pregunta sería algo así: ¿cuáles son las implicaciones educacionales y políticas de que haya algunos conocimientos con significados generalizables, y un grado de objetividad, que no pueden ser reducidos a sus contextos u orígenes? Las implicaciones son: concluir si hay motivos suficientes para negar acceso a tales conocimientos a la próxima generación, independientemente de sus backgrounds sociales y culturales.

\section{Referencias bibliográficas}

Abbott, A. (2001). Chaos of disciplines. Chicago: Chicago University Press.

Barnett, M. (2006). Vocational knowledge and vocational pedagogy. En M. Young y J. Gamble (eds.). Knowledge, curriculum and qualifications for South African further education. Pretoria: HSRC Press.
Bernstein, B. (2000). Pedagogy, symbolic control, and identity: Theory, research, critique. Oxford: Rowman \& Littlefield.

Charlot, B. (2009). School and the pupils' work. Sísifo. Educational Sciences Journal, 10, 87-94. Recuperado de http://sisifo.fpce.ul.pt.

Christie, F. y Maton, K. (de próxima aparición, 2011). Disciplinarity: Functional Linguistics and Sociological Perspectives. Londres: Continuum.

Durkheim. E. (1983). Pragmatism and sociology (J. C. Whitehouse, trad.). Cambridge: Cambridge University Press.

Gibbons, M.; Limoges, C.; Schwartzman, S.; Nowotny, H.; Trow, M. y Scott, P. (1994). The new production of knowledge: The dynamics of science and research in contemporary societies. Londres: Sage.

Gove, M. (2009). Failing schools need new leadership. Recuperado de http://michaelgovemp.typepad.com/files/ gove-2009-conference-speech-2.pdf.

Kronman, A. (2007). Education's end: Why our colleges and universities have given up on the meaning of life. New Haven: Yale University Press.

Marrero, A. (2008). Hermione en Hogwarts o sobre el éxito escolar de las niñas. Mora (B. Aires), 14(1), 29-42. Recuperado de: http://www.scielo.org.ar/scielo. php?script=sci_arttext $\&$ pid $=$ S1853001X2008000100 002\&lng=es\&nrm=iso

Moore, R. (2004). Education and society: Issues and explanations in the sociology of education. Londres: Polity Press.

Moore, R. (2011). Making the break: Disciplines and disciplinarity. En K. Christie y K. Maton (2011).

Muller, J. (2011). Through other's eyes; the fate of disciplines. En K. Christie y K. Maton (2011).

Oates, T. (2009). Missing the point: Identifying a wellgrounded common core. Cambridge: Cambridge Assessment.

White, J. (2007). What schools are for and why? Impact, 14. Recuperado de http://www.philosophy-of education. org/impact/impact_details.asp?id=14

Yates, L. \& Young, M. (2010). Globalisation, knowledge and the curriculum. European Journal of Education, 45(1), 4-10.

Young, M. (1971). Knowledge and control: New directions for the sociology of education. Londres: Collier-Macmillan.

Young, M. (1998). The curriculum of the future. Londres: Routledge. 
Young, M. (2008). Bringing knowledge back. En From social constructivism to social realism in the sociology of education. Londres: Routledge.

Young, M. (2009a). Education, globalisation and the 'voice of knowledge'. Journal of Education and Work, 22(3), 193-204.

Young, M. (2009b). What are schools for? En H. Daniels, J. Lauder y J. Porter (eds.). Knowledge, values and educational policy (pp. 10-18). Londres: Routledge.
Young, M. (2010). Alternative educational futures for a knowledge society. European Educational Research Journal, 9(1), 1-12.

Young, M. (2011). Why educators must differentiate knowledge from experience. Pacific Asian Education, 22(1).

Young, M. y Muller, J. (2010). Three educational scenarios for the future: Lessons from the sociology of knowledge. European Journal of Education, 45(1), 11-27. 